\title{
LESSONS LEARNT FROM TWO DECADES OF GRADUATE TRACER RESEARCH: RECOMMENDATIONS FOR THE SOUTH AFRICAN CONTEXT
}

\author{
J. Senekal ${ }^{*}$ \\ e-mail: janine.senekal@gmail.com
}

\author{
N. Munro* \\ e-mail: munron@ukzn.ac.za \\ *Discipline of Psychology \\ School of Applied Human Sciences \\ University of KwaZulu-Natal \\ Durban, South Africa
}

\section{ABSTRACT}

Graduate tracer studies may be an appropriate research method for responding to various problems in the South African higher education context, including difficulties associated with higher education transformation and graduate employability. However, there is little contextrelevant literature on the implementation of the various methodologies that may be used, and no assessment of the relevance of these methods for the South African context. In an attempt to synthesise graduate tracer study research, and to recommend potential models for conducting graduate tracer studies in South Africa, a systematic quantitative literature review was conducted of 23 graduate tracer studies from 13 countries, published between 1995 and 2016 . The findings from this review point to three potential models for implementation in the South African context: a large-scale model, a smaller-scale model, and a mixed-method model. These recommended models may allow for the more efficient and effective implementation of graduate tracer studies across the South African context.

Keywords: graduate tracer studies, alumni research, systematic quantitative literature review, career destinations, employment trends

\section{INTRODUCTION}

With the increased national focus on higher education transformation and graduate employability, many higher education institutions may wish to conduct empirical research to measure the extent of and change related to transformation and graduate employability. Graduate tracer studies may be considered a suitable option for this, yet there is little contextrelevant literature assessing different methodological options. Institutions may utilise methodologies that are not appropriately efficient or effective for their context, resulting in 
implementation difficulties and even poor-quality results. There is a need to identify suitable models as a starting point for institutions and researchers who wish to conduct graduate tracer studies in the South African context.

In different contexts, graduate tracer studies may be known by other names, including graduate destination surveys, alumni research, or tracer studies. Although these studies may differ in subtle ways, they all utilise the same strategy of using higher education alumni as a source of educational evaluation and for information about employment behaviour postgraduation (Du Toit et al. 2014; Shongwe and Ocholla 2011). In this article, the term "graduate tracer study" will be assumed to include those studies also referred to as "graduate destination studies" or "alumni research", taking place after graduation which focus on alumni employment destinations, rather than "exit" studies and similar evaluation-only surveys.

Graduate tracer studies provide information regarding typical career trajectories as well as higher education programme relevance and development, based on the reflections and evaluations of the educational experiences and employment activities of alumni from higher education institutions (Aina and Moahi 1999; De Guzman and de Castro 2008; Mubuuke, Businge and Kiguli-Malwadde 2014). Importantly, graduate tracer studies can be distinguished from graduate opinion or exit surveys which, in the South African context, typically survey alumni at the point of graduation (Cape Higher Education Consortium (CHEC) 2013). Several South African higher education institutions conduct graduate opinion surveys at their graduation ceremonies, and these surveys typically "aim to gather a quick 'snapshot' of job search behaviour, employment status, entry-level salaries, as well as satisfaction with the higher education institution, the curriculum they offer and its relevance to the workplace" (CHEC 2013, 7). Although useful for illuminating short-term trends, "exit studies" do not provide a longitudinal view of higher education graduates' employment and their workplace activities. Until the CHEC's (2013) Pathways from University to Work publication (which traced graduates from four universities, two years after their graduation), "graduate destination research [has been] highly underdeveloped [in South Africa] ... and there is no systematic attempt to understand graduate pathways outside of a few sporadic institutionally-based surveys" (CHEC 2013, 7). The bulk of graduate tracer studies seems to have taken place in Australia, the United Kingdom (UK) and the United States of America (USA).

For several social, political and economic reasons (e.g. slow economic growth, youth unemployment, increased access to higher education), understanding the employability and (un)employment of South African university graduates has become increasingly important (Rogan et al. 2015, vii). Implementing regular graduate tracer studies by higher education institutions and/or relevant higher education organisations could facilitate identifying long-term 
trends in employment and the workplace productivity of higher education graduates (CHEC 2013); and assist in linking these trends to the aforementioned social, political and economic dynamics. Moreover, Kraak (2015) argues that the results from graduate tracer studies are being increasingly used on a global scale to provide nuanced information about graduate employment, career pathways and the graduate labour market. It is thus probable that the South African higher education and labour market systems would also benefit from the regular collection of context-specific data of this kind.

In addition to informing higher education programme relevance and development, the information provided by graduate tracer studies may also inform the career decisions of prospective and current students, and assist these students in conceptualising realistic and shortto medium-term career trajectories in the contemporary labour market. Parents, career counsellors, and school and university teachers could also make use of the findings from graduate tracer studies in their guidance and teaching of prospective and current students (Goldwyn-Simpkins 2015). Graduate tracer studies may also contribute to a higher education institution's corporate profile where institutional "success" markers (e.g. flagship programmes with high graduate employment and occupational success rates) could be used for marketing purposes. However, graduate tracer studies may also be conceptualised as part of a global neoliberal agenda. Within this agenda, there is a focus on quantifiable outcomes, privatisation, and individual institutional success where higher education institutions become the marketplace of the knowledge economy (Maistry 2012). Overall, however, graduate tracer studies could also allow higher education institutions to be "more accountable, efficient, and aligned with public needs" (Cabrera, Weerts and Zulick 2005, 13).

Du Toit et al. $(2014,854)$ highlight that graduate tracer studies are complex because they aim to evaluate multiple domains of "university teaching and qualifications ... [and] different pathways from study to work, further study, [and] future aspirations" on a longitudinal basis. As a result, the methods used for a graduate tracer study are of critical importance. However, relatively little has been written about the methodological trends and difficulties associated with conducting graduate tracer studies. Therefore, a systematic quantitative literature review of existing graduate tracer studies was identified as necessary, in order to propose potential methods for conducting graduate tracer studies in the South African context (Petticrew and Roberts 2006).

It is apparent that South African higher education institutions would benefit from the implementation of regular graduate tracer studies in order to identify long-term trends in employment and employability. As such, this systematic quantitative literature review aimed to collate how graduate tracer studies have been conducted in order to recommend methods for 
the South African context. This study addressed the following research questions:

1. What are the purposes of graduate tracer studies?

2. What methods, sampling techniques and data analysis techniques have been used to conduct graduate tracer studies?

3. In terms of population and sample access in the South African context, which methods might be recommended for conducting graduate tracer studies?

\section{METHODOLOGY}

\section{Research design}

A systematic quantitative literature review methodology was used for this study (protocol reference HSS/0830/015H), and this involved "[k]nowledge creation [through] ... systematically searching for existing literature using key search words to identify papers for inclusion, based on clearly articulated and reproducible criteria" (Pickering et al. 2015, 1761). Systematic quantitative literature reviews sit on a continuum between narrative literature reviews and meta-analyses and are important for bridging the gap between what is already known and what is still unknown, including the gap between research and practical implementation (Thomas and Harden 2008, Pickering et al. 2015). In the case of this systematic quantitative literature review (subsequently referred to as the systematic review), the "known" were the existing graduate tracer study methods used mainly in the USA, UK and Australia, and the "unknown" is how to implement these methods in both independent and coordinated ways in the South African context. Petticrew and Roberts $(2006,21)$ identify that one of the reasons to conduct a systematic review of "past methodological research is ... to promote the development of new methodologies".

\section{Search procedure and inclusion criteria}

Keywords specific to graduate tracer study research were identified, and used to search for relevant literature through the public domain of the online journal database EBSCO host, and from higher education organisations, such as the Cape Higher Education Consortium, Graduate Careers Australia (GCA), Higher Education Careers Services Unit, and the Association of African Universities (AAU). Keywords included a combination of "graduate destination", "alumni", and "tracer", as well as "study", "research", and "survey" in a multiple search strategy. New terms were added to the keyword searches as they were identified, including "longitudinal surveys". Bibliographies of journal articles were also used to identify further relevant articles, organisations, and institutions to search for more literature. 
Inclusion and exclusion criteria determined which of the accessed graduate tracer studies would be included in this systematic review. Elements from the "PICOS" acronym (i.e. participants, intervention, comparisons undertaken, outcomes, and study design), that are typically used to guide the inclusion of research articles in a systematic review (Crisp 2015) were adapted for the purposes of this study. As Crisp (2015) notes, not all PICOS elements are relevant for non-intervention reviews, and therefore it was only required that the graduate tracer study articles specified their participants, outcomes, and study design as a first set of inclusion criteria. Specifically, the participants of the graduate tracer study should have been graduates from a university (thereby excluding graduates from technical and vocational education, and secondary education graduates). The literature should also have included an evaluation of the educational programmes and/or tracking of alumni's further education and employment activity after graduation. The study design should have used some form of a graduate tracer study (quantitative, qualitative or mixed method) in that it provided the above outcomes with the above participants. Furthermore, because the research questions primarily pertained to research methods and not research findings, there was less stringent focus on appraising and excluding studies based on their data quality and findings.

Further inclusion criteria for the review included publication dates: between 1995 and 2016. This time frame was selected to accommodate the trend of the massification and diversification of global higher education systems over the past two decades (Dawson, Charman and Kilpatrick 2013). Moreover, it was only possible to include articles/literature published in English. Further exclusion of literature took place based on the information provided in the article. For example, some publications referred to research from organisations such as GCA and AAU, yet did not contain the actual methods used to conduct the original research. In total, 23 publications were selected for inclusion in this systematic review, including seven from South Africa, three from the USA, two each from Australia and Uganda, and single studies from Botswana, Canada, Ghana, India, Indonesia, New Zealand, Philippines and the UK.

\section{Analysis of literature}

Using the aforementioned inclusion criteria (specifically participants, outcomes, and study design), the included studies were analysed in terms of the participants (i.e., target population), outcomes (i.e., tracking employment/further study and/or educational evaluation), and study design (i.e., type of method, type of data analysis, size of sampling frame, response rate, sample source, and data collection strategy). To facilitate an enhanced contextual analysis, country type was also included in the analytic strategy. To aid systematic identification, the data for this systematic review was extracted using a table (see Table 1). 
Before analysing the extracted information, the information (data) was simplified and coded to facilitate "organising, quantifying and analysing [the] data" (Robson 2011, 266). The coding was done manually by each author. Each research study was coded as a combination of three "clusters" of purposes (see Table 1, column 3). Cluster A (studies numbered in Table 1 from 1 to 6 ) included studies with an employment (i.e. tracking alumni's employment status) purpose, while Cluster B (studies numbered in Table 1 from 7 to 16) included studies with an employment and evaluation (of higher education courses/programmes or experiences) purpose. Cluster $\mathrm{C}$ studies (studies numbered in Table 1 from 17 to 23 ) included those with an employment focus and which included a specific agenda (which was often linked to policy changes or market related relevance), which may also include an evaluation purpose.

The target population from each research article was coded as being from a single department (necessarily within a single higher education institution), a single institution-wide population, or a population from across multiple higher education institutions. These target populations were then sub-coded by their focus (i.e., short-term [tracking graduates from within the last two years] or long-term [including and tracking graduates from multiple years, and generally beyond two years]).

The type of method was coded as being quantitative, qualitative, or mixed methods. A separate code was created for studies that were mainly quantitative but included some openended questions (i.e. not fully mixed method in approach). Types of data analysis were linked to the type of method, and were coded as descriptive statistics only, descriptive statistics with some basic comparisons, or descriptive and inferential statistics. Some of the mainly quantitative studies which included some open-ended questions also included "keyword searches" in their analysis of the open-ended questions responses, and therefore "keyword searches" features as a particular type of analysis for these studies. A separate qualitative analysis code was also included.

The country of origin of each research study was coded according to the United Nations Development Programme's (UNDP) (2015) Human Development Index (HDI). Specifically, the categorisations of very high, high, medium, low, and very low HDIs were used. The HDI provides a crude measure of human development (i.e. opportunities for life choices and capabilities) across dimensions of life expectancy, education, and standard of living. The authors reasoned that these dimensions of human development were intricately linked to graduate career trajectories and destinations. Although no categorisation fully reflects the complex social, economic and development dynamics within a country (McFarlane 2006), it was deemed important to reflect these socio-economic dynamics even if at a superficial level. Given the intended use of this systematic review (i.e. to propose an optimal method for graduate tracer studies in South Africa), what was deemed effective in certain countries with a very high 
HDI may not be feasible for South African graduate tracer studies. The UNDP (2015) classifies South Africa as having a medium HDI.

The sizes of the sampling frame were coded as being small ( $0-1000)$, medium ( 1000 $10000)$, large (10 000-100 000), or very large (100 000+), and response rates were coded as very low (0-24\%), low (25-45\%), medium (46-65\%), or high (66-100\%). The source of the sample and data collection methods varied greatly, and so no coding was used for these categories.

Descriptive analysis using frequencies was conducted for the codes under each variable. Further descriptive analysis using frequency tables was used to compare each category to every other category to identify possible trends in the data. No inferential statistics were conducted, due to the descriptive nature of the data as well as the small sample size ( $n=23$ ), so the findings are suggestive, not conclusive. To enhance the reliability of this systematic review, the two authors independently conducted the abovementioned search strategies, and reviewed each study separately in terms of the inclusion and exclusion criteria (Littell 2005).

\section{FINDINGS AND DISCUSSION}

The findings pertaining to the purposes of graduate tracer studies and the methods and techniques used are presented in Table 1, and then discussed. The discussion of the table is aligned to the research questions, reflecting purposes (question 1), methods and techniques (question 2), and recommended methods for the South African context (question 3). Thereafter, a discussion of the interaction between the tracer study methods and techniques and the purposes of graduate tracer studies is presented. The resultant relevance of the findings for the South African context is discussed, followed by the proposed models for the South African context.

\section{Purposes of graduate tracer studies}

Of the 23 studies, 13 were published after 2010, with the remaining 10 studies being published between 1998 and 2010. Eight of the studies were from countries with a very high HDI, while 12 of the studies arose from countries with a medium HDI. The remaining three studies came from countries with a low HDI. As per the inclusion criteria, all 23 studies had the purpose of tracking (un)employment trends in graduates. Six of the studies focused exclusively on tracking (un)employment trends in graduates (Cluster A). Ten of the studies included an evaluation of higher education programmes (Cluster B) in addition to the employment tracking. Seven of the studies (Cluster C) included a specific agenda (relating to policy development or market related relevance) over and above the employment and evaluation purpose. 
Table 1: Summary of the 23 graduate tracer studies

\begin{tabular}{|c|c|c|c|c|c|c|c|c|c|c|}
\hline & $\begin{array}{c}\text { Author(s) and } \\
\text { Year }\end{array}$ & \begin{tabular}{|c|} 
Purpose \\
\end{tabular} & $\begin{array}{c}\text { Target } \\
\text { population }\end{array}$ & Method & Analysis & HDI & $\begin{array}{l}\text { Sample } \\
\text { frame }\end{array}$ & $\begin{array}{l}\text { Response } \\
\text { rate }{ }^{d}\end{array}$ & Sample source & Data collection \\
\hline 1 & Cable (2013) & \multirow{6}{*}{ 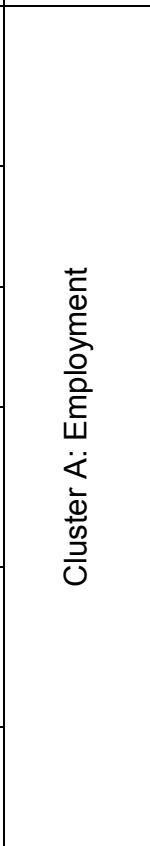 } & $\begin{array}{c}\text { Multiple } \\
\text { institutions - long } \\
\text { term }\end{array}$ & $\begin{array}{c}\text { Quan, some } \\
\text { open-ended } \\
\text { questions }\end{array}$ & $\begin{array}{c}\text { Descriptive, } \\
\text { Basic } \\
\text { comparisons, } \\
\text { Keyword searches }\end{array}$ & $\begin{array}{c}\text { New Zealand } \\
\text { (Very high) }\end{array}$ & $\begin{array}{c}130 \\
\text { Small }\end{array}$ & $\begin{array}{l}42 \% \\
\text { Low }\end{array}$ & $\begin{array}{l}\text { University } \\
\text { records and } \\
\text { Linkedln }\end{array}$ & $\begin{array}{l}\text { Survey assumed } \\
\text { - format not } \\
\text { specified }\end{array}$ \\
\hline 2 & \begin{tabular}{|l} 
Cape Higher \\
Education \\
Consortium (2013)
\end{tabular} & & $\begin{array}{c}\text { Multiple } \\
\text { institutions - long } \\
\text { term }\end{array}$ & Quan & $\begin{array}{c}\text { Descriptive, } \\
\text { Basic } \\
\text { comparisons } \\
\end{array}$ & $\begin{array}{l}\text { South Africa } \\
\text { (Medium) }\end{array}$ & $\begin{array}{l}24710 \\
\text { Large }\end{array}$ & $\begin{array}{l}22.5 \% \\
\text { Very low }\end{array}$ & $\begin{array}{l}\text { University } \\
\text { records }\end{array}$ & $\begin{array}{l}\text { Online survey } \\
\text { and telephonic } \\
\text { reminders }\end{array}$ \\
\hline 3 & $\begin{array}{l}\text { Higher Education } \\
\text { Statistics Agency } \\
(2013)\end{array}$ & & $\begin{array}{c}\text { Multiple } \\
\text { institutions - short } \\
\text { term }\end{array}$ & Quan & $\begin{array}{l}\text { Descriptive, } \\
\text { Basic } \\
\text { comparisons }\end{array}$ & UK (Very high) & \begin{tabular}{|l}
567490 \\
Very large
\end{tabular} & $\begin{array}{l}72 \% \\
\text { High }\end{array}$ & $\begin{array}{l}\text { University } \\
\text { records }\end{array}$ & $\begin{array}{c}\text { Survey assumed } \\
\text { - format not } \\
\text { specified }\end{array}$ \\
\hline 4 & $\begin{array}{l}\text { Letseka, Cosser, } \\
\text { Breier and Visser } \\
(2010)\end{array}$ & & $\begin{array}{c}\text { Multiple } \\
\text { institutions - short } \\
\text { term }\end{array}$ & Quan & $\begin{array}{l}\text { Descriptive and } \\
\text { inferential } \\
\text { statistics }\end{array}$ & $\begin{array}{l}\text { South Africa } \\
\text { (Medium) }\end{array}$ & $\begin{array}{l}14195 \\
\text { Large }\end{array}$ & $\begin{array}{c}15 \% \\
\text { Very low }\end{array}$ & $\begin{array}{l}\text { National higher } \\
\text { education } \\
\text { database } \\
\text { (HEMIS) }\end{array}$ & $\begin{array}{l}\text { Survey assumed } \\
\text { - format not } \\
\text { specified }\end{array}$ \\
\hline 5 & $\begin{array}{l}\text { Mouton, Boshoff, } \\
\text { James and Treptow } \\
(2010)\end{array}$ & & $\begin{array}{c}\text { Multiple } \\
\text { institutions - short } \\
\text { to long term }\end{array}$ & Quan & $\begin{array}{l}\text { Descriptive and } \\
\text { inferential } \\
\text { statistics }\end{array}$ & $\begin{array}{l}\text { South Africa } \\
\text { (Medium) }\end{array}$ & Un-known & $\begin{array}{l}\text { Unknown } \\
(12064 \\
\text { completed } \\
\text { surveys) }\end{array}$ & $\begin{array}{l}\text { University } \\
\text { records }\end{array}$ & Online survey \\
\hline 6 & $\begin{array}{l}\text { National Association } \\
\text { of Colleges and } \\
\text { Employers (2016) }\end{array}$ & & $\begin{array}{c}\text { Multiple } \\
\text { institutions - short } \\
\text { term }\end{array}$ & Quan & $\begin{array}{c}\text { Descriptive, Basic } \\
\text { comparisons }\end{array}$ & $\begin{array}{l}\text { USA (Very } \\
\text { high) }\end{array}$ & $\begin{array}{l}468250 \\
\text { Very large }\end{array}$ & $\begin{array}{l}62.3 \% \\
\text { Medium }\end{array}$ & $\begin{array}{l}\text { University } \\
\text { records and } \\
\text { Linkedln }\end{array}$ & $\begin{array}{l}\text { Survey - format } \\
\text { not specified }\end{array}$ \\
\hline 7 & $\begin{array}{l}\text { Aina and Moahi } \\
\text { (1999) }\end{array}$ & \multirow{4}{*}{ 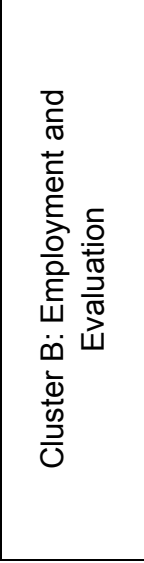 } & $\begin{array}{c}\text { Single department } \\
\text { - short to long } \\
\text { term }\end{array}$ & Quan & $\begin{array}{l}\text { Descriptive, } \\
\text { Basic } \\
\text { comparisons }\end{array}$ & $\begin{array}{l}\text { Botswana } \\
\text { (Medium) }\end{array}$ & $\begin{array}{c}187 \\
\text { Small }\end{array}$ & $\begin{array}{l}41.2 \% \\
\text { Low }\end{array}$ & \begin{tabular}{|c|} 
University \\
records and \\
current students' \\
network \\
\end{tabular} & $\begin{array}{l}\text { Postal survey } \\
\text { and reminders }\end{array}$ \\
\hline 8 & Carroll (2015) & & $\begin{array}{c}\text { Multiple } \\
\text { institutions - long } \\
\text { term } \\
\end{array}$ & Quan & $\begin{array}{l}\text { Descriptive, } \\
\text { Basic } \\
\text { comparisons } \\
\end{array}$ & $\begin{array}{c}\text { Australia (Very } \\
\text { high) }\end{array}$ & 91492 & $\begin{array}{c}19.7 \% \\
\text { Very low }\end{array}$ & $\begin{array}{l}\text { University } \\
\text { records }\end{array}$ & Online survey \\
\hline 9 & Gotch (2008) & & $\begin{array}{c}\text { Single institution - } \\
\text { short term }\end{array}$ & $\begin{array}{c}\text { Quan, some } \\
\text { open-ended } \\
\text { questions }\end{array}$ & $\begin{array}{c}\text { Descriptive, } \\
\text { Basic } \\
\text { comparisons } \\
\text { Keyword searches }\end{array}$ & $\begin{array}{l}\text { USA (Very } \\
\text { high) }\end{array}$ & $\begin{array}{c}3888 \\
\text { Medium }\end{array}$ & $\begin{array}{l}27 \% \\
\text { Low }\end{array}$ & $\begin{array}{l}\text { University } \\
\text { records }\end{array}$ & $\begin{array}{l}\text { Online and } \\
\text { postal survey } \\
\text { and postal } \\
\text { reminders }\end{array}$ \\
\hline 10 & Guthrie (2014) & & \begin{tabular}{|c|} 
Multiple \\
institutions - short \\
term
\end{tabular} & Quan & $\begin{array}{c}\text { Descriptive, } \\
\text { Basic } \\
\text { comparisons } \\
\end{array}$ & $\begin{array}{c}\text { Australia (Very } \\
\text { high) }\end{array}$ & \begin{tabular}{|c|}
253887 \\
Very large
\end{tabular} & $\begin{array}{c}55 \% \\
\text { Medium }\end{array}$ & $\begin{array}{l}\text { University } \\
\text { records }\end{array}$ & $\begin{array}{c}\text { Survey in person } \\
\text { (online and } \\
\text { telephonic) }\end{array}$ \\
\hline
\end{tabular}




\begin{tabular}{|c|c|c|c|c|c|c|c|c|c|c|}
\hline & $\begin{array}{c}\text { Author(s) and } \\
\text { Year }\end{array}$ & $\underset{\mathrm{a}}{\text { Purpose }}$ & $\begin{array}{c}\text { Target } \\
\text { population } b\end{array}$ & Method & Analysis & HDI & $\begin{array}{l}\text { Sample } \\
\text { frame }^{c}\end{array}$ & $\begin{array}{l}\text { Response } \\
\text { rate }{ }^{d}\end{array}$ & Sample source & Data collection \\
\hline 11 & $\begin{array}{l}\text { Mubuuke and Kiguli- } \\
\text { Malwadde (2014) }\end{array}$ & & $\begin{array}{c}\text { Single department } \\
\text { - short and long } \\
\text { term }\end{array}$ & Quan & $\begin{array}{c}\text { Descriptive, Basic } \\
\text { comparisons }\end{array}$ & Uganda (Low) & $\begin{array}{c}90 \\
\text { Small }\end{array}$ & $\begin{array}{l}80 \% \\
\text { High }\end{array}$ & $\begin{array}{l}\text { University } \\
\text { records }\end{array}$ & Email survey \\
\hline 12 & $\begin{array}{l}\text { North Carolina State } \\
\text { University (2015) }\end{array}$ & & $\begin{array}{l}\text { Single institution - } \\
\text { long term }\end{array}$ & Quan & $\begin{array}{c}\text { Descriptive and } \\
\text { inferential } \\
\text { statistics }\end{array}$ & $\begin{array}{l}\text { USA (Very } \\
\text { high) }\end{array}$ & $\begin{array}{l}14019 \\
\text { Large }\end{array}$ & $\begin{array}{l}18.5 \% \\
\text { Low }\end{array}$ & \begin{tabular}{|c|} 
University \\
records and US \\
postal service \\
records \\
\end{tabular} & $\begin{array}{l}\text { Online survey } \\
\text { and postal and } \\
\text { email reminders }\end{array}$ \\
\hline 13 & $\begin{array}{l}\text { Shongwe and } \\
\text { Ocholla (2011) }\end{array}$ & & $\begin{array}{c}\text { Single department } \\
\text { - long term }\end{array}$ & $\begin{array}{l}\text { Quan, } \\
\text { some open- } \\
\text { ended } \\
\text { questions }\end{array}$ & $\begin{array}{c}\text { Descriptive, } \\
\text { Basic } \\
\text { comparisons, } \\
\text { Keyword searches }\end{array}$ & $\begin{array}{l}\text { South Africa } \\
\text { (Medium) }\end{array}$ & $\begin{array}{l}125 \\
\text { Small }\end{array}$ & $\begin{array}{l}40 \% \\
\text { Low }\end{array}$ & \begin{tabular}{|c|} 
University \\
records, and \\
snowball, visiting \\
employment/ \\
residence \\
\end{tabular} & $\begin{array}{l}\text { Postal survey } \\
\text { and personal } \\
\text { follow up }\end{array}$ \\
\hline 14 & Sing (2010) & & $\begin{array}{c}\text { Single department } \\
\text { - long term }\end{array}$ & Qual & Qual methods & $\begin{array}{l}\text { South Africa } \\
\text { (Medium) }\end{array}$ & $\begin{array}{c}11 \\
\text { Small } \\
\end{array}$ & $\mathrm{n} / \mathrm{a}$ & $\begin{array}{l}\text { University } \\
\text { records }\end{array}$ & Interviews \\
\hline 15 & Stilwell (2004) & & $\begin{array}{l}\text { Single department } \\
- \text { long term }\end{array}$ & Mixed & $\begin{array}{c}\text { Descriptive, } \\
\text { Basic } \\
\text { comparisons, } \\
\text { Keyword searches }\end{array}$ & $\begin{array}{l}\text { South Africa } \\
\text { (Medium) }\end{array}$ & $\begin{array}{c}100 \\
\text { Small }\end{array}$ & $\begin{array}{l}40 \% \\
\text { Low }\end{array}$ & $\begin{array}{l}\text { University } \\
\text { records }\end{array}$ & $\begin{array}{l}\text { Postal survey } \\
\text { and email } \\
\text { reminder }\end{array}$ \\
\hline 16 & $\begin{array}{l}\text { Zembere and } \\
\text { Chinyama (1998) }\end{array}$ & & $\begin{array}{l}\text { Single institution - } \\
\text { long term }\end{array}$ & Quan & $\begin{array}{l}\text { Descriptive, } \\
\text { Basic } \\
\text { comparisons }\end{array}$ & Malawi (Low) & $\begin{array}{c}2000 \\
\text { Medium }\end{array}$ & $\begin{array}{l}29.4 \% \\
\text { Low }\end{array}$ & $\begin{array}{c}\text { Radio and } \\
\text { newspaper } \\
\text { adverts, } \\
\text { employer and } \\
\text { city records }\end{array}$ & Postal survey \\
\hline 17 & $\begin{array}{l}\text { De Guzman and de } \\
\text { Castro (2008) }\end{array}$ & 흥 $\frac{\pi}{0}$ & $\begin{array}{c}\text { Single institution - } \\
\text { long term } \\
\text { (market/policy } \\
\text { needs) } \\
\end{array}$ & Quan & $\begin{array}{l}\text { Descriptive and } \\
\text { inferential } \\
\text { statistics }\end{array}$ & $\begin{array}{l}\text { Philippines } \\
\text { (Medium) }\end{array}$ & $\begin{array}{c}5000 \\
\text { Medium }\end{array}$ & $\begin{array}{c}54 \% \\
\text { Medium }\end{array}$ & $\begin{array}{l}\text { University } \\
\text { records }\end{array}$ & $\begin{array}{c}\text { Postal and email } \\
\text { survey }\end{array}$ \\
\hline 18 & $\begin{array}{l}\text { Hayati and } \\
\text { Muschlish (2013) }\end{array}$ & 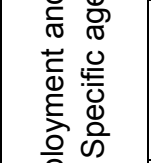 & $\begin{array}{c}\text { Single institution - } \\
\text { short term } \\
\text { (relevance to } \\
\text { market/field) } \\
\end{array}$ & Quan & Descriptive & $\begin{array}{l}\text { Indonesia } \\
\text { (Medium) }\end{array}$ & $\begin{array}{l}288 \\
\text { Small }\end{array}$ & $\begin{array}{c}18.4 \% \\
\text { Very low }\end{array}$ & $\begin{array}{l}\text { University } \\
\text { records }\end{array}$ & $\begin{array}{c}\text { Survey assumed } \\
\text { - format not } \\
\text { clear }\end{array}$ \\
\hline 19 & $\begin{array}{l}\text { Jaakkimainen, } \\
\text { Schultz, Glazier, } \\
\text { Abrahams and } \\
\text { Verma (2012) }\end{array}$ & 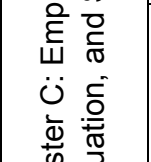 & $\begin{array}{c}\text { Single department } \\
\text { - long term } \\
\text { (impact of policy } \\
\text { changes) }\end{array}$ & Quan & $\begin{array}{c}\text { Descriptive and } \\
\text { inferential } \\
\text { statistics }\end{array}$ & $\begin{array}{l}\text { Canada (Very } \\
\text { high) }\end{array}$ & $\begin{array}{l}857 \\
\text { Small }\end{array}$ & $\begin{array}{l}100 \% \\
\text { High }\end{array}$ & $\begin{array}{l}\text { University } \\
\text { records }\end{array}$ & $\begin{array}{c}\text { n/a } \\
\text { Using existing } \\
\text { information }\end{array}$ \\
\hline 20 & \begin{tabular}{|l} 
Lassey, Lassey and \\
Boamah (2013)
\end{tabular} & 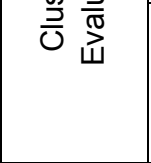 & $\begin{array}{c}\text { Single department } \\
- \text { long term } \\
\text { (Employment in } \\
\text { Ghana) }\end{array}$ & Quan & $\begin{array}{l}\text { Descriptive and } \\
\text { inferential } \\
\text { statistics }\end{array}$ & $\begin{array}{l}\text { Ghana } \\
\text { (Medium) }\end{array}$ & $\begin{array}{c}354 \\
\text { Small }\end{array}$ & $\begin{array}{c}99.7 \% \\
\text { High }\end{array}$ & $\begin{array}{l}\text { University } \\
\text { records }\end{array}$ & $\begin{array}{l}\text { Interview and } \\
\text { telephonic follow } \\
\text { up }\end{array}$ \\
\hline
\end{tabular}




\begin{tabular}{|c|c|c|c|c|c|c|c|c|c|c|}
\hline & $\begin{array}{c}\text { Author(s) and } \\
\text { Year }\end{array}$ & $\underset{\mathrm{a}}{\text { Purpose }}$ & $\begin{array}{c}\text { Target } \\
\text { population } b\end{array}$ & Method & Analysis & HDI & $\begin{array}{l}\text { Sample } \\
\text { frame }\end{array}$ & $\begin{array}{c}\text { Response } \\
\text { rate }^{d}\end{array}$ & Sample source & Data collection \\
\hline 21 & $\begin{array}{l}\text { Lutwama and } \\
\text { Kigongo-Bukenya } \\
(2004)\end{array}$ & & $\begin{array}{c}\text { Single department } \\
- \text { long term } \\
\text { (relevance of } \\
\text { education to } \\
\text { market/field) }\end{array}$ & Qual & Qual methods & Uganda (Low) & $\begin{array}{c}60 \text { selected } \\
\text { from } 217 \\
\text { Small }\end{array}$ & $\mathrm{n} / \mathrm{a}$ & $\begin{array}{l}\text { Renowned LIS } \\
\text { lecturers, } \\
\text { employers, } \\
\text { stakeholders, } \\
\text { other graduates }\end{array}$ & Focus groups \\
\hline 22 & $\begin{array}{l}\text { Rogan, Reynolds, } \\
\text { du Plessis, Bally } \\
\text { and Whitfield (2015) }\end{array}$ & & $\begin{array}{c}\text { Multiple } \\
\text { institutions - long } \\
\text { term (post- } \\
\text { apartheid labour } \\
\text { market) } \\
\end{array}$ & Quan & $\begin{array}{l}\text { Descriptive and } \\
\text { inferential } \\
\text { statistics }\end{array}$ & $\begin{array}{l}\text { South Africa } \\
\text { (Medium) }\end{array}$ & $\begin{array}{c}4927 \\
\text { Medium }\end{array}$ & $\begin{array}{l}43 \% \\
\text { Low }\end{array}$ & $\begin{array}{l}\text { University } \\
\text { records }\end{array}$ & $\begin{array}{l}\text { Online survey } \\
\text { and telephonic } \\
\text { interview follow }\end{array}$ \\
\hline 23 & Varalakshmi (2006) & & $\begin{array}{c}\text { Single department } \\
\text { - long term } \\
\text { (environmental } \\
\text { relevance) }\end{array}$ & $\begin{array}{c}\text { Quan, some } \\
\text { open-ended } \\
\text { questions }\end{array}$ & $\begin{array}{l}\text { Descriptive, } \\
\text { Basic } \\
\text { comparisons } \\
\text { Keyword, } \\
\text { searches }\end{array}$ & India (Medium) & $\begin{array}{c}30 \\
\text { Small }\end{array}$ & $\begin{array}{l}90 \% \\
\text { High }\end{array}$ & Not specified & Postal survey \\
\hline
\end{tabular}

a Purpose code: Cluster A: "employment" = tracking alumni employment patterns; Cluster B: "employment" and "evaluation" = course/study evaluation; Cluster C: "employment", and/or "evaluation", and "specific agenda" = relating to an additional specified purpose of study

b Target population code: "long term" = over more than 2 years after graduation, including multiple graduation years; "short term" = within 2 years of graduation, only those 2 years of graduates

c Sample frame: Sampling frame size codes: small 0-1000, medium: 1000-10000, large: 10000-100000, very large: 100000+

desponse rate codes: very low $=0-24 \%$, low $=25-45 \%$, medium $=46-65 \%$, high $=66-100 \%$ 


\section{Methods and techniques of graduate tracer studies}

Nine studies were conducted using a population from a single higher education institution department, with seven of these having a purely long-term focus (i.e. multiple graduation years beyond two years). The remaining two had a short- to/and long-term focus. Five studies had a population from a single higher education institution, three with a long-term focus, and two with a short-term focus (i.e. tracking graduates within two years of graduation). The remaining nine had populations from multiple institutions: four with a long-term focus, four with a shortterm focus, and one with both a short- and long-term focus.

Among the 23 studies reviewed, 18 relied upon university records as the primary sample source. Within these 18 studies, four supplemented university records with other methods. Four studies used only "other methods" when sourcing their sample, which included snowball sampling (e.g. through current students' knowledge of alumni's contact details), LinkedIn, online professional registration systems (trainee information and ongoing records), national databases of graduates, personal visits to places of employment or residence, and radio or newspaper advertisements. Varalakshmi (2006) did not specify where their sample was sourced from.

The sizes of the sampling frames varied from 30 to over 500 000. Small sampling frames (under 1000 ) were reported for 11 of the studies, while four studies reported medium sized sampling frames (between $1000-10$ 000). Four studies reported large sampling frames (from 10000-100000) and three studies had very large sampling frames (greater than 100 000). One study did not have an identifiable sampling frame size.

Five studies had very low response rates (0-24\%), seven had low response rates $(25-$ $45 \%$ ), three had medium response rates (46-65\%) and five had high response rates (66-100\%). Response rates were not reported (or could not be calculated) for three of the studies, two of which selected participants from a larger sample for participation in a focus group or interview. Of the two studies with 100 per cent response rates, one used existing available data, and both targeted very specific populations (for example, medical graduates), allowing ease of access to every participant.

Among the 23 studies reviewed, 20 used a survey or questionnaire with rating scales, and categorical or short answer questions. The use of surveys in these studies generated quantitative data. Of these 20 studies, four studies included a few open-ended questions in the surveys. Only Stilwell (2004) used mixed methods, including a survey component, while two studies used qualitative methods. Lutwama and Kigongo-Bukenya (2004) relied on a focus group method, while Sing (2010) used individual in-depth interviews. Jaakkimainen et al. (2012) used existing information as a source of information about the selected population, and was classified as a 
quantitative study.

As mentioned above, data was collected via a survey method in most of the studies, some specifying these surveys as online, telephonic or postal. Nine of the 20 studies reported using a combination of survey methods, however it is likely that several others used a combination, but did not specify this. Three studies reported using an online survey only, while another three reported only using a postal survey. Of these 20 studies, five sent reminders to complete the survey through email, post or telephonically.

Analysis methods used in the studies under review mirror the method type. Of the 20 quantitative studies, one used only descriptive statistics, eight used descriptive statistics with basic comparisons, and seven used descriptive and inferential statistics. All four of the quantitative studies with some open-ended questions additionally used keyword searches in the analysis. The mixed method study included in this review used descriptive statistics with basic comparisons and keyword searches. The two qualitative studies used qualitative analysis methods, one referring to using a "heuristic process of phenomenological analysis" (Lutwama and Kigongo-Bukenya 2004, 103), while the other only noted that "qualitative data analysis was applied to the answers obtained" (Singh 2010, 41).

\section{Interaction of purposes, methods and techniques of graduate tracer studies}

There are notable patterns in the relationships between the studies' purposes, target populations, sampling size frames and response rates. Four of the five studies with high response rates (66$100 \%$ ) had populations from a single department with a long-term focus, while the remaining study with a high response rate targeted graduates from multiple institutions, but had a shortterm focus. Although not all nine studies with single department target populations secured high response rates, all of them had sampling frame sizes of less than 1000 . Four of the five multiple institution studies that only included a short-term focus had sampling frames greater than 100 000, the only exception being the South African multiple institution (short-term focus) study (Letseka et al. 2010,5) which had a sampling frame of 14 195. Higher response rates therefore appear to be associated with target populations from single departments with a longterm focus and small sampling frame (less than 1000 ), or to populations from multiple institutions with a short-term focus, and very large sampling frames (over 100000 ).

Considerations across the purpose of the study, the target population, and the country of publication also yielded notable trends. Only one of the nine studies (Rogan et al. 2015) with populations from multiple institutions included a specific agenda, while the rest only focused on employment, and may have also included an analysis of degree/programme/institution evaluation trends. Similarly, six of the seven studies (all except Rogan et al. 2015) which 
included a specific agenda had populations from a single higher education institution (four in a single department, and two across a single university). This affirms the abovementioned trend that studies with specific agendas tend not to rely on larger sampling frames which appear to be purposed towards employment and evaluation only. Studies with smaller sampling frames and single department populations were mostly published in countries with medium and low HDIs, while countries with very high HDIs produced studies with the largest sampling frames and with populations from multiple institutions.

There is a spread of response rates associated with the different methods used to source samples in the studies under review. As such, it is not evident which strategy might be most effective for sourcing samples. Based on response rates, there is no clear indication as to which data collection methods might be regarded as most effective. All studies published after 2010 used either online surveys or a combination of survey methods with reminders; and postal-only survey methods were only used prior to 2010. This suggests that as technology advances, a greater variety of methods are being used to collect data in graduate tracer studies. All the postal-only surveys were conducted in countries with low to medium HDIs, which affirms the likely slower rate of technology adoption and integration in these countries (UNDP 2015, 7).

\section{Relevance to South African context}

The seven South African graduate tracer studies under review are likely to have the greatest relevance as they arise from the context within which the authors wish to make recommendations. Despite highly differential levels of human and socio-economic development in South Africa, the country remains a developing nation (Cline and Luiz 2013; Schonfeldt, Gibson and Vermeulen 2010). The other studies under review from low to medium HDI countries are also relevant to the South African context based on aggregated similarities in labour market and human development conditions. South African graduate tracer studies are likely to benefit from considering differential development levels across regions and provinces, in terms of how these play out in the higher education and labour market, and what this may imply for methodology. Importantly, graduate tracer studies from high HDI countries are not irrelevant in the South African context, but rather provide insight into the possibilities that exist for tracer studies if conducted on a regular basis and as information systems, infrastructure and development are enhanced.

\section{Methodological relevance}

Of the 21 studies where response rates are relevant, 12 of these had response rates under 50 per cent. Although these response rates may make generalisability of findings difficult, response 
rates are not necessarily reflective of sample representativeness, which may be more important in terms of generalisability (Lambert and Miller 2014, 38). Low response rates bring the question of non-response patterns into question, as the sample may be biased in some way if there are significant differences between those who did respond and those who did not. However, if the sample is representative of the population, the sample is less likely to be biased, as the differences between those who did and did not respond may be random.

To address the issue of low response rates, a possible solution is to implement two research phases by selecting a subsample from respondents in the initial phase for qualitative interviews or focus group discussions (Purcell and Elias 2004, 4). This phased strategy would increase access and response rates for the second phase of the research, as a confirmed list of correct contact details would exist, and potentially willing respondents could be identified from respondents from the first phase. Another way to address low response rates is to use existing data and records, for example, by accessing business networking sites (such as LinkedIn), professional organisations (such as the Health Professions Council in the South African context), or further training and internship records (NACE 2016). Drawing in the aforementioned types of data sources could enhance the accuracy of graduate contact and employment details, without needing to rely only on university records and survey responses.

The regular and systematic implementation of graduate tracer studies within a higher education institution or across a group of institutions was recommended by various studies to allow for tracking medium- to long-term trends (CHEC 2013; Hayati and Muschlish 2013; NACE 2016; Purcell and Elias 2004). In order for this to happen, there needs to be "buy-in" from the relevant higher education institution and all departments involved, to support the implementation of graduate tracer studies and ensure their efficacy (Hayati and Muschlish 2013). It was also noted that initial employment destinations (within a year of graduation) do not necessarily reflect later career decisions, as many graduates have false starts in their careers, change career paths altogether or go on to further study (Hobden and Hobden, 2015, 8). Therefore, while short-term focused studies allow for the evaluation of courses, longer term studies are needed for identifying long-term employment behaviour.

Higher education organisations may play an important part in coordinating and implementing graduate tracer studies across multiple higher education institutions, as seen in some of the studies under review. The use of these organisations to oversee the research process involved in conducting graduate tracer studies means that there is less work for each higher education institution to do, as they are only responsible for distributing surveys to their graduates. This strategy is similar to what was done in several of the South African studies under review, and could be further enhanced and implemented in the future. The coordinated 
efforts of higher education institutions and organisations would produce accurate and recent records about higher education and employment in South Africa. With the results of such graduate tracer studies being made available to the public and for use by other researchers, this would increase the need and desire to produce higher quality data.

\section{Data collection efficiency}

The lack of up-to-date higher education institution records limits the efficiency and efficacy of graduate tracer studies. If higher education institutions furnish outdated or missing graduate contact details, this limits the access and representativeness of the studies. It would be beneficial to identify which method of contact is most reliable for making initial contact with graduates as part of conducting graduate tracer studies. While it was unclear which method of data collection was most effective, it is likely that a combination of survey distribution methods would be the most effective, including sending reminders. Initial contact could be used to confirm correct contact details, with the actual survey being distributed based on those verified contact details. However, research is needed to identify which methods are better than others, and what makes those methods more efficient. Response rates appear to be linked to data collection methods and sample access; however, further research is needed to identify whether they are predictors of response rates.

\section{Proposed models for the South African context}

To reflect the diversity of development across South African provinces and cities (and the subsequent diversity of public higher education institutions in South Africa), three models of graduate tracer studies are proposed. In the developing provinces and regions of South Africa or Historically Disadvantaged Institutions (where funding, resources and infrastructure are compromised), graduate tracer studies are more likely to be successful (e.g. ease of access to target population, reliable sampling frame, high response rates) with a population from a single department or higher education institution, with a sample size of less than 1000 . Ideally, a longterm population (i.e. two-years after graduation) should be targeted, and the study should include a graduate employment component, with the possible inclusion of a specific agenda and/or a programme evaluation component. Examples of relevant specific agendas could include tracing proportions of health care graduates from specific institutions (or regions) that stay in public (and perhaps rural) health care after community service and why. These kinds of studies could either be qualitative and/or quantitative, with corresponding analysis methods.

In South African provinces or regions that are relatively more developed, it is suggested that graduate tracer studies include a population across multiple higher education institutions, 
with a short-term focus employment activity, potentially including some evaluation. Due to the larger population, quantitative methods and corresponding analysis methods would be feasible. These larger-scale graduate tracer studies are likely to be successful if coordinated by higher education organisations (e.g. the Council on Higher Education, the Centre for Higher Education Transformation, and the Cape Higher Education Consortium). A third method may combine the above two suggested methods. A two-phase graduate tracer study could be conducted, with an initial quantitative phase of a large population, followed by a qualitative phase of a smaller population addressing a specific issue.

For sample access, higher education records should be utilised, alongside alternative methods, including social media, current student knowledge, and further professional registration. Higher education institutions should make efforts to ensure the quality of their alumni records, to aid accessing the alumni population easier. When using a survey for data collection, a combination of postal, email, and telephonic communication is recommended. While this is not verified as being more effective, it increases that chances of more comprehensive access.

\section{CONCLUSION}

In this systematic review, 23 graduate tracer studies met our inclusion criteria, and were reviewed and collated in order to propose potential methods for conducting graduate tracer studies in the South African context. Studies were accessed from the public domain, and were included based on participants, outcomes and study design, as well as being published in English and within a 20-year time frame. Information was extracted from the articles using a grid system, relating to the nature of each study, focusing particularly on the methods used, target population and purpose. Although the disparate populations and context of the studies under review compromised direct comparisons, the review facilitated the recommendation of three potential models for South African graduate tracer research, and this is an important bridge towards further research in this area. The proposed models could be implemented to test their relevance, efficiency and efficacy, which could lead to the development and regular implementation of graduate tracer studies across South African higher education institutions. An inclusion of both long- and short-term foci would allow for the identification of mediumto long-term trends in education and employment in South Africa.

Research around response rates and population representativeness would be beneficial for higher education research in general. Consolidation of the connections between South African higher education institutions and organisations is also important, in order to ensure up-to-date alumni records, and to ensure that the implementation of graduate tracer studies is easier in the 
future. These three recommended models may be used to aid South African institutions and researchers in their endeavours to conduct graduate tracer studies more efficiently and effectively, and assist them in monitoring and assessing the plethora of higher education issues, towards transforming this central sector of South African society.

\section{REFERENCES}

Aina, L. O. and K. Moahi. 1999. Tracer study of the Botswana library school graduates. Education for Information 17(3): 215-244.

Cable, D. A. J. 2013. The career experiences of industrial and organisational psychology graduates. New Zealand Journal of Psychology 42(1): 5-12.

Cabrera, A. F., D. J. Weerts and B. J. Zulick. 2005. Making an impact with alumni surveys. New Directions for Institutional Research (126): 5-17.

Carroll, D. 2015. Beyond graduation 2014: A report of graduates' work and study outcomes three years after course completion. Melbourne: Graduate Careers Australia Ltd.

Cape Higher Education Consortium. 2013. Pathways from university to work: A graduate destination survey of the 2010 cohort of graduates from the Western Cape universities. Wynberg: Cape Higher Education Consortium.

CHEC see Cape Higher Education Consortium.

Cline, G. B. and J. M. Luiz. 2013. Information technology systems in public sector health facilities in developing countries: The case of South Africa. BMC Medical Informatics and Decision Making 13(13): 1-13.

Crisp, B. R. 2015. Systematic reviews: A social work perspective. Australian Social Work 68(3): 284295. doi: 10.1080/0312407X.2015.1024266.

Dawson, P., K. Charman and S. Kilpatrick. 2013. The new higher education reality: What is an appropriate model to address the widening participation agenda? Higher Education Research and Development 32(5): 706-1721. doi: 10.1080/07294360.2013.776520.

De Guzman, A. B. and B. V. de Castro. 2008. Employment and employability profile of a select group of Filipino college graduates. KEDI Journal of Educational Policy 5(1): 63-81.

Du Toit, J., A. Kraak, J. Favish and L. Fletcher. 2014. From study to work: Methodological challenges of a destination survey in the Western Cape, South Africa. Assessment \& Evaluation in Higher Education 39(7): 853-864. doi: 10.1080/02602938.2013.875118.

Goldwyn-Simpkins, H. 2015. What do graduates do. Manchester: Prospects/AGCAS.

Gotch, C. 2008. Results of the survey of 2005-2006 alumni. https://ir.wsu.edu/documents/ 2017/01/alumni-survey-undergraduate-class-of-2005-06-full-report.pdf (Accessed 21 May 2015).

Guthrie, B. 2014. Australian graduate survey 2013: A report of the conduct of the 2013 Australian graduate survey. Melbourne: Graduate Careers Australia Ltd.

Hayati, N. R. and T. I. Muschlish. 2013. Studies for enhancing graduates' relevance and competitiveness. Review of Higher Education and Self-Learning 6(20): 33-40.

Higher Education Statistics Agency. 2013. "Destinations of leavers from higher education 2011/12". HESA.ac.uk. https://www.hesa.ac.uk/data-and-analysis/publications/destinations-2011-12 (Accessed 7 April 2015).

Hobden, P. and S. Hobden. 2015. A study of the transition pathways of school level scholarship recipients into work and tertiary education. South African Journal of Education 35(3): 1-10.

Jaakkimainen, R. L., S. E. Schultz, R. H. Glazier, C. Abrahams and S. Verma. 2012. Tracking family medicine graduates. Where do they go, what services do they provide and whom do they see? BMC Family Practice 13(26): 1-11. doi: 10.1186/1471-2296-13-26. 
Kraak, A. 2015. The value of graduate destination survey data in understanding graduate unemployment: A focus on the universities of technology. South African Journal of Labour Relations 39(1): 93-113.

Lambert, A. D. and A. L. Miller. 2014. Lower response rates on alumni surveys might not mean lower response representativeness. Educational Research Quarterly 37(3): 38-51.

Lassey, A. T., P. D. Lassey and M. Boamah. 2013. Career destination of University of Ghana medical school graduates of various year groups. Ghana Medical Journal 47(2): 87-91.

Letseka, M., M. Cosser, M. Breier and M. Visser. 2010. Student retention \& graduation destination: Higher education and labour market access and success. Cape Town: HSRC Press.

Littell, J. H. 2005. Lessons from a systematic review of effects of multisystemic therapy. Children and Youth Services Review 27(4): 445-463. doi:10.1016/j.childyouth.2004.11.009

Lutwama, E. and I. M. N. Kigongo-Bukenya. 2004. A tracer study of the East African school of library and information science graduates 1995-1999 working in Uganda. SA Journal of Library and Information Science 70(2): 99-109.

Maistry, S. M. 2012. Confronting the neo-liberal brute: Reflections of a higher education middle-level manager. South African Journal of Higher Education 26(3): 515-528.

McFarlane, C. 2006. Crossing borders: Development, learning and the North-South divide. Third World Quarterly 27(8): 1413-1437.

Mouton, J., N. Boshoff, M. James and R. Treptow. 2010. Consensus study on the state of the humanities in South Africa: Status, prospects and strategies. Pretoria: Academy of Science of South Africa.

Mubuuke, A. G., F. Businge and E. Kiguli-Malwadde. 2014. Using graduates as key stakeholders to inform training and policy in health professions: The hidden potential of tracer studies. African Journal of Health Professions Education 6(1): 52-55. doi: 10.7196/AJHPE.302.

NACE see National Association of Colleges and Employers.

National Association of Colleges and Employers. 2016. First destinations for the college class of 2015. Bethlehem, PA: National Association of Colleges and Employers.

North Carolina State University. 2015. 2015 alumni survey: Introduction, methods, and alumni demographic profile. https://oirp.ncsu.edu/wordpress/wp-content/uploads/2015/12/alum15. intro_.pdf (Accessed November 21, 2016).

Petticrew, M. and H. Roberts. 2006. Systematic reviews in the social sciences: A practical guide. New Jersey: Wiley-Blackwell.

Pickering, C., J. Grignon, R. Steven, D. Guitart and J. Byrne. 2015. Publishing not perishing: How research students transition from novice to knowledgeable using systematic quantitative literature reviews. Studies in Higher Education 40(10): 1756-1769. doi: 10.1080/03075079.2014.914907.

Purcell, K. and P. Elias. 2004. Seven years on: Graduate careers in a changing labour market. Manchester: HECSU, ESRC, IER, ESRU.

Robson, C. 2011. Surveys and questionnaires. In Real world research: A resource for users of social research methods in applied settings, 235-277. Chichester, West Sussex: Wiley.

Rogan, M., J. Reynolds, U. du Plessis, R. Bally and K. Whitfield. 2015. Pathways through university and into the labour market. Report on a graduate tracer study from the Eastern Cape. http://www.lmip.org.za/sites/default/files/documentfiles/LMIP\%20Report\%2018\%20Eastern\%2 0Cape\%20WEB_0.pdf (accessed April 18, 2017).

Schonfeldt, H. C., N. Gibson and H. Vermeulen. 2010. The possible impact of inflation on nutritionally vulnerable household in a developing country using South Africa as a case study. Nutrition Bulletin 35: 254-267.

Shongwe, M. and D. N. Ocholla. 2011. A tracer study of LIS graduates from the University of Zululand, 2000-2009. Mousaion 29(2): 277-245.

Sing, R. 2010. "Career trajectories of masters in education (MEd) Class of 1999 at the University of the Witwatersrand" masters research report, the University of the Witwatersrand, 2010, $0010619 \mathrm{~T}$. 
Stilwell, C. 2004. Alumni perceptions of post-graduate information and library science education programme at the University of Natal, South Africa. South African Journal of Libraries and Information Science 70(1): 20-29. Doi: http://dx.doi.org/10.7553/70-1-691

Thomas, J. and A. Harden. 2008. Methods for the thematic synthesis of qualitative research in systematic reviews. BMC Medical Research Methodology 8(45): 1-10. doi: 10.1186/1471-2288-8-45

UNDP see United Nations Development.

United Nations Development Programme. 2015. Human development report 2015. New York: United Nations Development Programme.

Varalakshmi, R. S. R. 2006. Education 21st century LIS professionals - Needs and expectations: A survey of Indian LIS professionals and alumni. Journal of Education for Library and Information Science 47(3): 181-199.

Zembere, S. N. and M. P. M. Chinyama. 1998. The University of Malawi graduate tracer study 1996. http://rc.aau.org/files/ZEMBERE.pdf 